\title{
Warnmeldungen zu Lebensmittelkontaktmaterialien im Europäischen Schnellwarnsystem RASFF von 2012 bis 2017
}

\section{Alert notifications concerning food contact material published in the Rapid Alert System for Food and Feed between 2012 and 2017}

\author{
Paula Kuchheuser $^{2} \cdot$ Katja Dünnebier $^{1}$ (D) Jan Hoffbauer ${ }^{1} \cdot$ Andreas Butschke $^{1} \cdot$ Marc Birringer $^{2}$
}

Received: 12 June 2018/Accepted: 23 August 2018/Published online: 18 September 2018

(C) The Author(s) 2018

\section{Zusammenfassung}

Das Europäische Schnellwarnsystem für Lebensmittel und Futtermittel (RASFF) dient europäischen Mitgliedstaaten als Instrument für einen schnellen Informationsaustausch über Gesundheitsrisiken durch Lebensmittel, Futtermittel und Lebensmittelkontaktmaterialien. Eine differenzierte Auswertung der übermittelten Meldungen zu Lebensmittelkontaktmaterialien über einen längeren Zeitraum lag bislang nicht vor. Daher wurde eine Analyse der zwischen 2012 und 2017 veröffentlichten Warnmeldungen, mit Fokus auf Art und Material betroffener Lebensmittelkontaktmaterialien sowie zugrundeliegende Gesundheitsrisiken, durchgeführt. Die Auswertung konzentriert sich explizit auf Warnmeldungen, welche im Schnellwarnsystem ein rasches Tätigwerden zuständiger Kontaktstellen erforderten und vorrangig behandelt wurden. Als häufige Gesundheitsrisiken erwiesen sich der Übergang von Metallionen, die Migration von primären aromatischen Aminen sowie die Migration von Ausgangsstoffen der Kunststoffherstellung aus Kontaktmaterialien in Lebensmittelsimulanzien. Die entsprechenden Warnmeldungen wurden hinsichtlich Art und Material der betroffenen Lebensmittelkontaktmaterialien sowie auf ursächliche Substanzen untersucht. Ein Übergang von Blei und Cadmium ließ sich häufig bei Gläsern, Bechern, Tassen und Geschirr aus dekoriertem Glas oder glasierter Keramik feststellen. Eine Migration von primären aromatischen Aminen wurde insbesondere bei Erzeugnissen aus Kunststoff oder Nylon nachgewiesen. Betroffen waren meist Pfannenwender oder Servierlöffel. Auch das Humankanzerogen 4,4'-Methylendianilin befand sich unter den migrierenden Substanzen. Eine Migration von Ausgangsstoffen der Kunststoffherstellung wie Melamin und Formaldehyd ließ sich vermehrt bei Lebensmittelkontaktmaterialien aus Melaminharz identifizieren, insbesondere bei Geschirr.

Schlüsselwörter Schnellwarnsystem · Lebensmittelkontaktmaterialien · Migration · Blei und Cadmium · Primäre aromatische Amine · Melamin und Formaldehyd

Katja Dünnebier

113@bvl.bund.de

$1 \quad$ Bundesamt für Verbraucherschutz und

Lebensmittelsicherheit (BVL), Abteilung 1

„Lebensmittelsicherheit“, Postfach 110260, 10832 Berlin,

Germany

2 Fachbereich Oecotrophologie, Hochschule Fulda-University

of Applied Sciences, Leipziger Straße 123, 36037 Fulda,

Germany 


\section{Abstract}

The European Rapid Alert System (RASFF) is a key tool for food safety used by EU member states for swift exchange about serious health risks due to food, feed and food contact materials. Until now, a detailed analysis of submitted notifications concerning food contact materials over a longer time period was not available. Therefore, an examination on alert notifications concerning food contact materials published between 2012 and 2017 was carried out. The focus of this analysis was on the type and the material of affected food contact materials as well as underlying health risks. This evaluation concentrates on alert notifications, which require quick action of the responsible contact points, and that were treated with priority. The release of metal ions as well as the migration of primary aromatic amines and of substances used in plastic manufacturing into food simulants are common health risks. Notifications about these health risks were examined for both type and material of food contact materials and causative substances. The release of lead and cadmium often occurred from glasses, cups, mugs and tableware made of painted glass or ceramic with decoration or glaze. The migration of primary aromatic amines was frequently identified with products made of plastic such as spatulas or serving spoons. In several cases, the human carcinogen 4,4'-methylenedianiline was among the migrating substances. The migration of starting substances used in plastic manufacturing like melamine and formaldehyde often appeared in food contact materials made of melamine resin, in particular from tableware.

Keywords Rapid Alert System for Food and Feed (RASFF) - Food contact material · Migration · Lead and cadmium . Primary aromatic amines $\cdot$ Melamine and formaldehyde

\section{Einleitung}

Das Europäische Schnellwarnsystem RASFF gilt als Schlüsselinstrument für Lebensmittel- und Futtermittelsicherheit in der Europäischen Union (EU) (European Commission 2009). Nach Maßgabe der Basis-Verordnung (Verordnung (EG) Nr. 178/2002) sowie der Durchführungs-Verordnung für das RASFF (Verordnung (EU) Nr. 16/2011) dient das RASFF zum Informationsaustausch über ernste Gesundheitsrisiken durch Lebensmittel, Futtermittel und Lebensmittelkontaktmaterialien. Neben der Europäischen Kommission, welche für die Administration des Meldesystems verantwortlich ist, besteht das Netzwerk aus Kontaktstellen in den Mitgliedstaaten der EU, den Mitgliedstaaten der Europäischen Freihandelsassoziation (EFTA) (European Commission o.J.), der EFTAÜberwachungsbehörde sowie der Europäischen Behörde für Lebensmittelsicherheit (EFSA). Auf nationaler Ebene übernimmt das Bundesamt für Verbraucherschutz und Lebensmittelsicherheit (BVL) die Zuständigkeiten der nationalen Kontaktstelle (BMEL 2016).

Ein Bestandteil des RASFF sind Schnellwarnmeldungen zu Lebensmittelkontaktmaterialien, welche gemäß ihrer Rahmen-Verordnung (Verordnung (EG) Nr. 1935/2004) Materialien und Gegenstände mit vorliegendem oder potenziellem Lebensmittelkontakt darstellen. Mangels ausführlicher Auswertungen wurde eine differenzierte Analyse der entsprechenden Warnmeldungen aus den Jahren 2012 bis 2017 vorgenommen. Das Ziel dieser vertiefenden Analyse bestand darin, risikobehaftete Erzeugnisse sowie vermehrt auftretende Gesundheitsrisiken zu identifizieren.

\section{Methoden}

Als Datenbasis der Analyse dienten die im interaktiven Online-Meldesystem iRASFF übermittelten Originalmeldungen sowie Folgemeldungen. Die Daten wurden anhand einer internen Datenbank des BVL über eingehende Schnellwarnmeldungen sowie Vergleichsdaten aus dem öffentlichen RASFF Portal der Europäischen Kommission validiert und im Anschluss für die Auswertung aufbereitet. Die Auswertung schließt keine Informations- und Grenzzurückweisungsmeldungen ein und konzentriert sich explizit auf Warnmeldungen, welche im Schnellwarnsystem ein rasches Tätigwerden zuständiger Kontaktstellen erfordern und vorrangig behandelt werden. Im Fokus der Auswertung standen sowohl Art und Material der Lebensmittelkontaktmaterialien als auch die den Meldungen zugrundeliegenden Gesundheitsrisiken.

\section{Ergebnisse}

Im Rahmen der Datenauswertung ließen sich vermehrt Warnmeldungen zum Übergang von Metallionen, zur Migration von primären aromatischen Aminen (paA) oder zur Migration von Ausgangsstoffen der Kunststoffherstellung bei Lebensmittelkontaktmaterialien identifizieren. In Tab. 1 sind europäische Rechtsvorschriften für Lebensmittelkontaktmaterialien sowie grundlegende europäische Rechtsvorschriften von Relevanz aufgeführt. 
Tab. 1 Übersicht europäischer Rechtsvorschriften für Lebensmittelkontaktmaterialien

\section{Allgemeine Rechtsgrundlagen für Lebensmittelkontaktmaterialien}

Verordnung (EG) Nr. 1935/2004 (Rahmen-Verordnung)*

des Europäischen Parlaments und des Rates vom 27. Oktober 2004 über Materialien und Gegenstände, die dazu bestimmt sind, mit Lebensmitteln in Berührung zu kommen und zur Aufhebung der Richtlinien 80/590/EWG und 89/109/EWG (ABl. L 338 vom 13.11.2004, S. 4)

Verordnung (EG) Nr. 2023/2006 (GMP-Verordnung)*

der Kommission vom 22. Dezember 2006 über gute Herstellungspraxis für Materialien und Gegenstände, die dazu bestimmt sind, mit Lebensmitteln in Berührung zu kommen (AB1. L 384 vom 29.12.2006, S. 75)

\section{Materialspezifische Rechtsgrundlagen für Lebensmittelkontaktmaterialien}

Verordnung (EU) Nr. 10/2011 (Kunststoff-Verordnung)*

der Kommission vom 14. Januar 2011 über Materialien und Gegenstände aus Kunststoff, die dazu bestimmt sind, mit Lebensmitteln in Berührung zu kommen (ABl. L 12 vom 15.1.2011, S. 1)

Verordnung (EG) Nr. 450/2009

der Kommission vom 29. Mai 2009 über aktive und intelligente Materialien und Gegenstände, die dazu bestimmt sind, mit Lebensmitteln in Berührung zu kommen (AB1. L 135 vom 30.05.2009, S. 3)

Verordnung (EG) Nr. 282/2008

der Kommission vom 27. März 2008 über Materialien und Gegenstände aus recyceltem Kunststoff, die dazu bestimmt sind, mit Lebensmitteln in Berührung zu kommen, und zur Änderung der Verordnung (EG) Nr. 2023/2006 (AB1. L 86 vom 28.03.2008, S. 9)

Richtlinie 84/500/EWG (Keramik-Richtlinie)*

Richtlinie des Rates vom 15. Oktober 1984 zur Angleichung der Rechtsvorschriften der Mitgliedstaaten über Keramikgegenstände, die dazu bestimmt sind, mit Lebensmitteln in Berührung zu kommen (84/500/EWG) (AB1. L 277 vom 20.10.1984, S. 12)

Richtlinie 2007/42/EG

Richtlinie 2007/42/EG der Kommission vom 29. Juni 2007 über Materialien und Gegenstände aus Zellglasfolien, die dazu bestimmt sind, mit Lebensmitteln in Berührung zu kommen (ABl. L 172 vom 30.06.2007, S. 71)

\section{Stoffspezifische Rechtsgrundlagen für Lebensmittelkontaktmaterialien}

Verordnung (EU) Nr. 284/2011*

der Kommission vom 22. März 2011 mit besonderen Bedingungen und detaillierten Verfahren für die Einfuhr von Polyamid- und Melamin-Kunststoffküchenartikeln, deren Ursprung oder Herkunft die Volksrepublik China bzw. die Sonderverwaltungsregion Hongkong, China, ist (ABl. L 77 vom 23.03.2011, S. 25)

Verordnung (EG) Nr. 1895/2005

der Kommission vom 18. November 2005 über die Beschränkung der Verwendung bestimmter Epoxyderivate in Materialien und Gegenständen, die dazu bestimmt sind, mit Lebensmitteln in Berührung zu kommen (AB1. L 302 vom 19.11.2005, S. 28)

Verordnung (EU) 2018/213

der Kommission vom 12. Februar 2018 über die Verwendung von Bisphenol A in Lacken und Beschichtungen, die dazu bestimmt sind, mit Lebensmitteln in Berührung zu kommen, und zur Änderung der Verordnung (EU) Nr. 10/2011 hinsichtlich der Verwendung dieses Stoffes in Lebensmittelkontaktmaterialien aus Kunststoff (ABl. L 41 vom 14.02.2018, S. 6)

Richtlinie 93/11/EWG

der Kommission vom 15. März 1993 über die Freisetzung von N-Nitrosaminen und N-nitrosierbaren Stoffen aus Flaschen- und Beruhigungssaugern aus Elastomeren oder Gummi (AB1. L 93 vom 17.04.1993, S. 37)

\section{Allgemeine Rechtsgrundlagen von Relevanz}

Verordnung (EG) Nr. 178/2002 (Basis-Verordnung)*

des Europäischen Parlaments und des Rates vom 28. Januar 2002 zur Festlegung der allgemeinen Grundsätze und Anforderungen des Lebensmittelrechts, zur Errichtung der Europäischen Behörde für Lebensmittelsicherheit und zur Festlegung von Verfahren zur Lebensmittelsicherheit (ABl. L 31 vom 01.02.2002, S. 1)

Verordnung (EG) Nr. 882/2004 (Kontroll-Verordnung)*

des Europäischen Parlaments und des Rates vom 29. April 2004 über amtliche Kontrollen zur Überprüfung der Einhaltung des Lebensmittel-und Futtermittelrechts sowie der Bestimmungen über Tiergesundheit und Tierschutz (AB1. L 165 vom 30.04.2004, S. 1)

Verordnung (EG) Nr. 1272/2008 (CLP-Verordnung)*

des Europäischen Parlaments und des Rates vom 16. Dezember 2008 über die Einstufung, Kennzeichnung und Verpackung von Stoffen und Gemischen, zur Änderung und Aufhebung der Richtlinien 67/548/EWG und 1999/45/EG und zur Änderung der Verordnung (EG) Nr. 1907/2006 (ABl. L 353 vom 31.12.2008, S. 1)

Verordnung (EU) Nr. 16/2011 (Durchführungs-Verordnung)* 
Tab. 1 (continued)

der Kommission vom 10. Januar 2011 mit Durchführungsbestimmungen für das Schnellwarnsystem für Lebens- und Futtermittel (ABl. L 6 vom 11.1.2011, S. 7)

Die Rechtstexte verstehen sich in der jeweils aktuellen Fassung und sind im offiziellen Portal für Rechtstexte der Europäischen Union abrufbar (www.eur-lex.europa.eu/). Vorschriften, die im Rahmen der hier vorgestellten Daten konkret zur Anwendung gekommen sind, sind mit * markiert

Tab. 2 Warnmeldungen zum Übergang von Metallionen bei Lebensmittelkontaktmaterialien im Zeitraum von 2012 bis 2017 (Anzahl an Warnmeldungen)

\begin{tabular}{|c|c|c|c|c|c|c|c|}
\hline \multicolumn{8}{|c|}{ Material der Produkte } \\
\hline Glas & $\begin{array}{l}\text { davon } \\
\text { dekoriert }\end{array}$ & Keramik & $\begin{array}{l}\text { davon mit } \\
\text { Dekoration/ } \\
\text { Glasur }\end{array}$ & $\begin{array}{l}\text { Metall/ } \\
\text { Legierung }\end{array}$ & $\begin{array}{l}\text { davon mit } \\
\text { Beschichtung }\end{array}$ & $\begin{array}{l}\text { davon mit } \\
\text { Holz, } \\
\text { Textilien }\end{array}$ & Warnmeldungen \\
\hline
\end{tabular}

\begin{tabular}{|c|c|c|c|c|c|c|c|c|}
\hline \\
\hline & $\begin{array}{l}\text { Art der Produkte } \\
\text { Utensilien zur Darreichung, } \\
\text { Aufnahme von } \\
\text { Lebensmitteln }\end{array}$ \\
\hline \multicolumn{9}{|l|}{$\begin{array}{l}\text { davon Gläser, Becher, } \\
\text { Tassen }\end{array}$} \\
\hline davon Geschirr & 1 & 1 & 15 & 15 & & & & 16 \\
\hline \multicolumn{5}{|l|}{ Utensilien zur Zubereitung } & 4 & 1 & 1 & 6 \\
\hline \multicolumn{5}{|l|}{ Kochgeschirr, Backgeschirr } & 1 & 1 & & 1 \\
\hline Summe & 26 & 25 & 23 & 20 & 5 & 2 & $\mathbf{1}$ & 54 \\
\hline
\end{tabular}

\section{1 Übergang von Metallionen bei Lebensmittelkontaktmaterialien}

Zwischen 2012 und 2017 sind 54 Warnmeldungen zum Übergang von Metallionen aus Lebensmittelkontaktmaterialien im RASFF veröffentlicht worden (Tab. 2). Bei insgesamt 164 übermittelten Warnmeldungen zu Lebensmittelkontaktmaterialien entspricht diese Anzahl einem Anteil von 32,9\%. Anhand der Tabelle ist erkennbar, dass sich die veröffentlichten RASFF-Meldungen zum Übergang von Metallionen beinahe ausnahmslos auf Lebensmittelkontaktmaterialien aus Glas oder Keramik beziehen (49 von 54 Meldungen; 90,7\%). In nahezu allen Fällen handelte es sich dabei um Erzeugnisse aus dekoriertem Glas (25 von 26 Meldungen) oder aus Keramik mit Dekoration oder Glasur (20 von 23 Meldungen). Weiterhin fällt auf, dass Lebensmittelkontaktmaterialien aus (dekoriertem) Glas insbesondere Gläser, Becher, Tassen darstellten (25 von 26 Meldungen; 96,2\%), während bei Lebensmittelkontaktmaterialien aus Keramik (mit Dekoration oder Glasur) häufig Geschirr betroffen war (15 von 23 Meldungen; 65,2\%). In Tab. 3 sind die nachgewiesenen Metallionen sowie die jeweils zu berücksichtigenden Grenzwerte bzw. zugrunde gelegten Beurteilungswerte aufgelistet.

Aus den betroffenen Lebensmittelkontaktmaterialien wurden mehrheitlich Übergänge der Metallionen Cadmium
$(\mathrm{Cd})$ und Blei $(\mathrm{Pb})$ aus Glas oder Keramik in Lebensmittelsimulanzien festgestellt. Während innerhalb des Zeitraums von 2012 bis 2017 lediglich vereinzelt Warnmeldungen zum Übergang von Aluminium (Al), Arsen (As), Cobalt (Co) und Zink ( $\mathrm{Zn})$ veröffentlicht wurden (15 von 49 Meldungen; 30,6\%), gab es deutlich mehr Meldungen über $\mathrm{Pb}$ (30 von 49 Meldungen; 61,2\%) sowie Cd (35 von 49 Meldungen; 71,4\%). Bei der Mehrzahl dieser Fälle ging es um den gleichzeitigen Übergang von $\mathrm{Pb}$ und $\mathrm{Cd}$ (26 von 49 Meldungen; 53,1\%), während lediglich 4 Meldungen zum alleinigen Übergang von $\mathrm{Pb}$ und 9 Meldungen zum alleinigen Übergang von $\mathrm{Cd}$ vorlagen. Bezogen auf die Gesamtzahl übermittelter Warnmeldungen stellen Meldungen zum Übergang von $\mathrm{Pb}$ einen Anteil von 18,3\% (30 von 164 Meldungen) und Meldungen zum Übergang von Cd einen Anteil von 21,3\% (35 von 164 Meldungen) dar.

Ein hoher Anteil der Warnmeldungen zum Übergang von $\mathrm{Pb}$ und $\mathrm{Cd}$ aus Glas oder Keramik in Lebensmittel basierte auf Messergebnissen in der Einheit $\mathrm{mg} /$ Stück $(\mathrm{Pb}$ in 20 Meldungen mit 56 Messwerten; Cd in 19 Meldungen mit 50 Messwerten), die auf ähnliche nationale Beurteilungsgrundlagen zurückzuführen sind. Von diesen insgesamt 21 Warnmeldungen berichteten 18 über einen gleichzeitigen Übergang von $\mathrm{Pb}$ und $\mathrm{Cd}$. Die Warnmeldungen betrafen ausnahmslos Lebensmittelkontaktmaterialien der Produktkategorie Gläser, Becher, Tassen, welche 
Tab. 3 Warnmeldungen zum Übergang von Blei und Cadmium bei Lebensmittelkontaktmaterialien aus Glas oder Keramik im Zeitraum 2012 bis 2017

\begin{tabular}{|c|c|c|c|c|c|c|}
\hline Einheit & Meldungen & Messwerte & MAX & MW & M & GW \\
\hline \multicolumn{7}{|l|}{ Blei (Pb) } \\
\hline \multicolumn{7}{|c|}{30 Meldungen von 49 Meldungen $^{1}, 97$ Messwerte } \\
\hline $\mathrm{mg} / \mathrm{dm}^{2}$ & 2 & 6 & 43 & 33,7 & 39,0 & 0,8 \\
\hline $\mathrm{mg} / \mathrm{L}$ & 3 & 15 & 73,7 & 39,16 & 45,40 & 4,0 \\
\hline $\mathrm{mg} /$ Stück & 20 & 56 & 399 & 46,88 & 18,20 & 2,0 \\
\hline $\mathrm{mg} /$ Stück & 5 & 20 & 169,62 & 38,65 & 12,69 & k. A. \\
\hline \multicolumn{7}{|c|}{ Cadmium (Cd) } \\
\hline \multicolumn{7}{|c|}{35 Meldungen von 49 Meldungen $^{1,2}, 94$ Messwerte } \\
\hline $\mathrm{mg} / \mathrm{dm}^{2}$ & 9 & 16 & 1,87 & 0,95 & 1,03 & 0,07 \\
\hline $\mathrm{mg} / \mathrm{L}$ & 2 & 4 & 5,44 & 4,21 & 4,27 & 0,3 \\
\hline $\mathrm{mg} / \mathrm{Stück}^{3}$ & 19 & 50 & 94,67 & 6,177 & 1,430 & 0,2 \\
\hline $\mathrm{mg} / \mathrm{Stück}^{3}$ & 6 & 24 & 1,126 & 0,505 & 0,453 & k. A. \\
\hline
\end{tabular}

$M A X$ Höchster Messwert, $M W$ Mittelwert, $M$ median, $G W$ Grenzwert bzw. zugrunde gelegter Beurteilungswert, $k$. A. keine Angabe

${ }^{1}$ Anzahl an Meldungen zum Übergang von Pb bzw. Cd bei insgesamt 49 Meldungen zum Übergang von Metallionen bei Lebensmittelkontaktmaterialien aus Glas oder Keramik (s. Tab. 2)

${ }^{2}$ Für eine Meldung zu einem Set von Lebensmittelkontaktmaterialien (Teller, Schüssel, Tasse) liegen Messwerte für die Schüssel in $\mathrm{mg} / \mathrm{L}$ und für den Teller in $\mathrm{mg} / \mathrm{dm}^{2}$ vor. Daher wird eine Meldung doppelt für Messergebnisse in verschiedenen Einheiten aufgeführt. Die Gesamtzahl der Meldungen beträgt jedoch 35 Stück

${ }^{3}$ In einzelnen Meldungen mit Messwerten in der Einheit mg/Stück wurden die zugrunde gelegten Grenzwerte nicht angegeben. Diese Meldungen wurden separat von Meldungen mit Messwerten und Grenzwerten in der Einheit $\mathrm{mg} /$ Stück dargestellt bis auf eine Ausnahme allesamt aus (dekoriertem) Glas bestanden. Die Warnmeldungen wurden vorwiegend durch die nationalen Kontaktstellen von Polen (9 von 21 Meldungen; 42,9\%) und Deutschland (6 von 21 Meldungen; $28,6 \%$ ), seltener von anderen europäischen Mitgliedstaaten übermittelt.

\subsection{Migration von primären aromatischen Aminen bei Lebensmittelkontaktmaterialien}

Bei insgesamt 164 übermittelten Warnmeldungen entsprechen die 50 Warnmeldungen, die zwischen 2012 und 2017 im RASFF über die Migration von primären aromatischen Aminen (paA) bei Lebensmittelkontaktmaterialien veröffentlicht wurden, einem Anteil von 30,5\% (Tab. 4). Die Auswertung der RASFF-Meldungen nach Art und Material der Produkte legte dar, dass sich Warnmeldungen zur Migration von paA vornehmlich auf Lebensmittelkontaktmaterialien aus Kunststoff (18 von 50 Meldungen; 36,0\%) oder Nylon (27 von 50 Meldungen; 54,0\%) beziehen. Andere Materialien waren eher selten beteiligt. In erster Linie waren Utensilien zur Zubereitung (27 von 50 Meldungen; 54,0\%) sowie Utensilien zur Darreichung und Aufnahme von Lebensmitteln (20 von 50 Meldungen; $40,0 \%$ ) betroffen. Hierbei liegt ein deutlicher Schwerpunkt bei Pfannenwendern (13 von 27 Meldungen; 48,1\%),
Servierlöffeln (8 von 20 Meldungen; 40,0\%) und Spaghettilöffeln (6 von 20 Meldungen; 30,0\%). Warnmeldungen zur Migration von paA bei Lebensmittelkontaktmaterialien aus Kunststoff oder Nylon gab es häufig zu Lebensmittelkontaktmaterialien aus China oder Hongkong (34 von 45 Meldungen; 75,6\%) und nur vereinzelt aus anderen, vorwiegend europäischen Ursprungsländer.

Eine hohe Anzahl an Meldungen lag zur Migration von paA als Stoffgruppe vor (26 von 45 Meldungen; 57,8\%). Häufig informierten Warnmeldungen sowohl über die Gesamtmigration von paA als auch die Migration zugehöriger Substanzen (Tab. 5). Demnach berichteten 31 Meldungen über die Migration von 4,4'-Methylendianilin (im Folgenden 4,4'-MDA) (31 von 45 Meldungen; 68,9\%), vornehmlich aus Nylon (22 von 31 Meldungen; 71,0\%). Deutlich weniger Meldungen gab es zur Migration von Anilin (9 von 45 Meldungen; 20,0\%), 4,4'-Bi-o-toluidin und 2,4-Toluoldiamin (im Folgenden TDA). In den Migrationsuntersuchungen wurden mitunter deutliche Überschreitungen der Nachweisgrenze für paA von $0,01 \mathrm{mg} / \mathrm{kg}$ festgestellt: Die höchsten Messwerte lassen sich bei der Gesamtmigration von paA bei Lebensmittelkontaktmaterialien aus Nylon sowie der Migration von 4,4'-MDA aus Kunststoff und aus Nylon identifizieren (Tab. 5). 
Tab. 4 Warnmeldungen zur Migration von primären aromatischen Aminen bei Lebensmittelkontaktmaterialien im Zeitraum von 2012 bis 2017 (Anzahl an Warnmeldungen)

\begin{tabular}{|c|c|c|c|c|c|c|}
\hline & \multicolumn{5}{|c|}{ Material der Produkte } & \multirow[t]{2}{*}{ Warnmeldungen } \\
\hline & Kunststoff $^{1}$ & $\begin{array}{l}\text { Metall/ } \\
\text { Legierung }\end{array}$ & Naturkautschuk & Nylon & $\begin{array}{l}\text { Papier/Pappe/ } \\
\text { Karton }\end{array}$ & \\
\hline \multicolumn{7}{|l|}{ Art der Produkte } \\
\hline $\begin{array}{l}\text { Utensilien zur Darreichung, Aufnahme von } \\
\text { Lebensmitteln }\end{array}$ & 6 & & 1 & 13 & & 20 \\
\hline davon Servierlöffel & 2 & & & 6 & & 8 \\
\hline davon Spaghettilöffel & 2 & & & 4 & & 6 \\
\hline Utensilien zur Zubereitung & 12 & 1 & & 14 & & 27 \\
\hline davon Pfannenwender & 5 & 1 & & 7 & & 13 \\
\hline Textilien zur Zubereitung, Darreichung ${ }^{2}$ & & & & & 3 & 3 \\
\hline Summe & 18 & $\mathbf{1}$ & $\mathbf{1}$ & 27 & 3 & $\mathbf{5 0}$ \\
\hline
\end{tabular}

${ }^{1}$ In die Kategorie „Kunststoff“ wurden alle Kunststoffmaterialien eingeordnet, die in den Meldungen nicht näher spezifiziert bzw. nicht eindeutig den Kategorien „Nylon“, „Melaminharz“, „Naturkautschuk“ oder „Silikonkautschuk“ zuzuordnen sind

${ }^{2}$ In die Kategorie der „Textilien zur Zubereitung und Darreichung“ zählten in der Auswertung Papierservietten und Platzdeckchen. In dieser Tabelle sind Meldungen zu farbigen und bedruckten Papierservietten aufgeführt

Tab. 5 Warnmeldungen zur Migration von primären aromatischen Aminen, 4,4'Methylendianilin und Anilin bei Lebensmittelkontaktmaterialien aus Kunststoff oder Nylon im Zeitraum 2012 bis 2017

\begin{tabular}{|c|c|c|c|c|c|c|c|}
\hline Material & Einheit & Meldungen & Messwerte & MAX & MW & M & $\mathrm{GW}^{1}$ \\
\hline \multicolumn{8}{|c|}{ Primäre aromatische Amine } \\
\hline \multicolumn{8}{|c|}{26 Meldungen $^{2}, 59$ Messwerte } \\
\hline Kunststoff & $\mathrm{mg} / \mathrm{kg}$ & 14 & 33 & 12,6217 & 2,6017 & 0,5630 & 0,01 \\
\hline Nylon & $\mathrm{mg} / \mathrm{kg}$ & 13 & 29 & 26,6 & 2,2545 & 0,1092 & 0,01 \\
\hline \multicolumn{8}{|c|}{ 4,4'-Methylendianilin } \\
\hline \multicolumn{8}{|c|}{31 Meldungen, 72 Messwerte } \\
\hline Kunststoff & $\mathrm{mg} / \mathrm{kg}$ & 9 & 21 & 34,2 & 5,1921 & 1,3178 & 0,01 \\
\hline Nylon & $\mathrm{mg} / \mathrm{kg}$ & 22 & 51 & 25,9 & 1,2659 & 0,0570 & 0,01 \\
\hline \multicolumn{8}{|l|}{ Anilin } \\
\hline \multicolumn{8}{|c|}{9 Meldungen, 17 Messwerte } \\
\hline Kunststoff & $\mathrm{mg} / \mathrm{kg}$ & 6 & 14 & 1,7215 & 0,3925 & 0,0242 & 0,01 \\
\hline Nylon & $\mathrm{mg} / \mathrm{kg}$ & 3 & 3 & 0,600 & 0,2084 & 0,0140 & 0,01 \\
\hline
\end{tabular}

\subsection{Migration von Ausgangsstoffen der Kunststoffherstellung bei Lebensmittelkontaktmaterialien}

Es gab 37 Warnmeldungen zur Migration von Ausgangsstoffen der Kunststoffherstellung (Monomere und andere Ausgangsstoffe, Zusatzstoffe und Hilfsstoffe), die zwischen 2012 und 2017 im RASFF bei Lebensmittelkontaktmaterialien veröffentlicht wurden, was bei insgesamt 164 übermittelten Warnmeldungen einem Anteil von 22,6\% entspricht (Tab. 6). Aus der Tabelle wird deutlich, dass die Warnmeldungen zur Migration von Ausgangsstoffen der Kunststoffherstellung mehrheitlich
Lebensmittelkontaktmaterialien aus Melaminharz auch in Kombination mit Beschichtung oder Bambus betrafen (21 von 37 Meldungen; 56,8\%). Dahingegen waren Lebensmittelkontaktmaterialien aus Kunststoff oder Naturkautschuk deutlich seltener Gegenstand einer Meldung. Bei den betroffenen Gegenständen handelte es sich häufig um Utensilien zur Darreichung und Aufnahme von Lebensmitteln (19 von 37 Meldungen; 51,4\%) - insbesondere um Geschirr (11 von 19 Meldungen; 57,9\%) - und seltener um Utensilien zur Zubereitung (5 von 37 Meldungen; 13,5\%). Weiterhin lagen vermehrt Warnmeldungen zur Migration von Ausgangsstoffen der Kunststoffherstellung bei Schraubdeckelgläsern (13 von 37 Meldungen; 35,1\%) vor (Tab. 6). 
Tab. 6 Warnmeldungen zur Migration von Ausgangsstoffen der Kunststoffherstellung bei Lebensmittelkontaktmaterialien im Zeitraum von 2012 bis 2017 (Anzahl an Warnmeldungen)

\begin{tabular}{|c|c|c|c|c|c|c|c|c|}
\hline & \multicolumn{7}{|c|}{ Material der Produkte } & \multirow[t]{2}{*}{ Warnmeldungen } \\
\hline & Glas & Kunststoff $^{1}$ & Melaminharz & $\begin{array}{l}\text { davon mit } \\
\text { Beschichtung }\end{array}$ & $\begin{array}{l}\text { davon } \\
\text { mit } \\
\text { Bambus }\end{array}$ & Naturkautschuk & unbekannt & \\
\hline \multicolumn{9}{|l|}{ Art der Produkte } \\
\hline $\begin{array}{l}\text { Utensilien zur Darreichung, } \\
\text { Aufnahme von } \\
\text { Lebensmitteln }\end{array}$ & & 1 & 16 & 10 & 1 & 1 & 1 & 19 \\
\hline davon Geschirr & & & 11 & 7 & & & & 11 \\
\hline $\begin{array}{l}\text { Utensilien zur } \\
\text { Aufbewahrung, } \\
\text { Konservierung }\end{array}$ & 13 & & & & & & & 13 \\
\hline $\begin{array}{l}\text { davon } \\
\quad \text { Schraubdeckelgläser }\end{array}$ & 13 & & & & & & & 13 \\
\hline Utensilien zur Zubereitung & & & 5 & & & & & 5 \\
\hline Summe & 13 & 1 & 21 & 10 & 1 & 1 & 1 & 37 \\
\hline
\end{tabular}

${ }^{1}$ In die Kategorie „Kunststoff“ wurden alle Kunststoffmaterialien eingeordnet, die in den Meldungen nicht näher spezifiziert bzw. nicht eindeutig den Kategorien „Nylon“, „Melaminharz“, „Naturkautschuk“ oder „Silikonkautschuk“ zuzuordnen sind

Tab. 7 Warnmeldungen zur Migration von Ausgangsstoffen der Kunststoffherstellung bei Lebensmittelkontaktmaterialien aus Melaminharz im Zeitraum von 2012 bis 2017 (Anzahl der Warnmeldungen)

\begin{tabular}{|c|c|c|c|c|c|c|}
\hline Jahr & 2012 & 2013 & 2014 & 2015 & 2016 & 2017 \\
\hline \multirow[t]{3}{*}{ Meldende Mitgliedstaaten } & DE: 4 & CY: 1 & \multirow[t]{3}{*}{-} & BE: 1 & DE: 1 & BE: 4 \\
\hline & LU: 2 & DE: 1 & & FR: 2 & FR: 1 & DE: 1 \\
\hline & LV: 1 & GR: 1 & & & & SI: 1 \\
\hline Meldungen zur Migration von Ausgangsstoffen der Kunststoffherstellung & 7 & 3 & 0 & 3 & 2 & 6 \\
\hline davon zu Lebensmittelkontaktmaterialien aus China oder Hongkong & 2 & 2 & 0 & 2 & 2 & 4 \\
\hline
\end{tabular}

$B E$ Belgien, $C Y$ Cypern, DE Deutschland, FR Frankreich, GR Griechenland, LU Luxemburg, LV Lettland, SI Slowenien

Die Hälfte der Warnmeldungen zur Migration von Ausgangsstoffen der Kunststoffherstellung bei Lebensmittelkontaktmaterialien aus Melaminharz betraf Lebensmittelkontaktmaterialien aus China oder Hongkong (12 von 21 Meldungen; $57,1 \%$ ). Seltener stammten die betroffenen Lebensmittelkontaktmaterialien aus anderen, vorwiegend europäischen Ursprungsländern. Die Auswertung der Meldungen nach dem Zeitpunkt der Übermittlung zeigt, dass in den Jahren 2012 bis 2017 unterschiedliche Anzahlen an Warnmeldungen zu diesem Sachverhalt übermittelt wurden (Tab. 7).

Aus den von Warnmeldungen betroffenen Lebensmittelkontaktmaterialien aus Melaminharz migrierten mehrheitlich Melamin (13 von 21 Meldungen; 61,9\%) oder Formaldehyd (15 von 21 Meldungen; 71,4\%). In mehr als einem Drittel der Meldungen wurde über die gleichzeitige Migration sowohl von Melamin als auch von Formaldehyd berichtet ( 8 von 21 Meldungen; 38,1\%). Eine Beurteilung der Messwerte zur Migration von Melamin erfolgte bis einschließlich 2012 anhand des Grenzwerts 30 mg/kg, ab
2013 anhand des Grenzwerts 2,5 mg/kg. Beide Substanzen zeigten hohe Migrationswerte: Eine Migration von Melamin ließ sich mit Messwerten von bis zu $273 \mathrm{mg} / \mathrm{kg}$ bei einem Grenzwert von $30 \mathrm{mg} / \mathrm{kg}$ nachweisen. Die Migration von Formaldehyd erreichte Konzentrationen von bis zu $770 \mathrm{mg} / \mathrm{kg}$ bei einem Grenzwert von $15 \mathrm{mg} / \mathrm{kg}$. Das entspricht einer 9-fachen Überschreitung des Grenzwerts für Melamin, während der Grenzwert für Formaldehyd um mehr als das 50-fache überschritten wird (Tab. 8).

\section{Diskussion}

\section{1 Übergang von Metallionen bei Lebensmittelkontaktmaterialien}

Warnmeldungen zum Übergang von Metallionen bei Lebensmittelkontaktmaterialien aus Glas oder Keramik beziehen sich beinahe ausnahmslos auf Erzeugnisse aus dekoriertem Glas oder aus Keramik mit Dekoration oder 
Tab. 8 Warnmeldungen zur Migration von Melamin und Formaldehyd bei

Lebensmittelkontaktmaterialien aus Melaminharz im Zeitraum 2012 bis 2017

\begin{tabular}{|c|c|c|c|c|c|c|}
\hline Einheit & Meldungen & Messwerte & MAX & MW & M & GW \\
\hline \multicolumn{7}{|c|}{ Melamin } \\
\hline \multicolumn{7}{|c|}{13 Meldungen, 32 Messwerte } \\
\hline $\mathrm{mg} / \mathrm{kg}$ & 5 & 8 & 273 & 198,99 & 212,99 & $30^{2}$ \\
\hline $\mathrm{mg} / \mathrm{kg}$ & 8 & 24 & 27,855 & 6,772 & 4,350 & $2,5^{3}$ \\
\hline \multicolumn{7}{|c|}{ Formaldehyd } \\
\hline \multicolumn{7}{|c|}{15 Meldungen, 23 Messwerte } \\
\hline $\mathrm{mg} / \mathrm{kg}$ & 15 & 23 & 770 & 219,77 & 114,37 & 15 \\
\hline
\end{tabular}

$M A X$ Höchster Messwert, $M W$ Mittelwert, $M$ median, $G W$ Grenzwert

${ }^{2}$ In Warnmeldungen angewandter Grenzwert bis einschließlich 2012

${ }^{3}$ In Warnmeldungen angewandter Grenzwert ab 2013
Glasur. Demnach beruht das zugrundeliegende Gesundheitsrisiko vermutlich nicht auf den Materialien Glas oder Keramik. Vielmehr ist zu vermuten, dass der Übergang von Metallionen auf die verwendete Dekoration oder Glasur zurückzuführen ist (Peltzer et al. 2015).

Warnmeldungen zum Übergang der Metallionen $\mathrm{Pb}$ und Cd bei Gläsern, Bechern, Tassen aus (dekoriertem) Glas wurden überwiegend durch die Kontaktstellen aus Polen und Deutschland übermittelt. Dass diese Mitgliedstaaten vermehrt meldeten, lässt sich womöglich auf die vorhandene Beurteilungsgrundlage auf nationaler Ebene zurückführen. Die Norm PN-B-13210:1997 des Polnischen Komitees für Normung und die Norm DIN 51032:2017-07 des Deutsches Instituts für Normung formulieren Beurteilungswerte für den Übergang von $\mathrm{Pb}$ und $\mathrm{Cd}$ bei Lebensmittelkontaktmaterialien aus Keramik, Glas und Glaskeramik. Da in der Keramik-Richtlinie keine Grenzwerte für spezifische Sachverhalte wie den Übergang von $\mathrm{Pb}$ und $\mathrm{Cd}$ aus dem Trinkrand definiert sind, dienen diese europaweit bekannten Normen sowohl in Polen und Deutschland als auch anderen Mitgliedstaaten für Lebensmittekontaktmaterialien aus Keramik. Sie sind Grundlage für die Beurteilung der Gesundheitsgefährdung gemäß Art. 3 der Rahmen-Verordnung. Die zugrunde gelegten Beurteilungswerte von $2,0 \mathrm{mg} \mathrm{Pb} / \mathrm{Stück}$ und $0,2 \mathrm{mg} \mathrm{Cd} / \mathrm{Stück}$ bei den Warnmeldungen beziehen sich auf den Übergang von Metallionen aus dem Trinkrand des untersuchten Lebensmittelkontaktmaterials.

Die Anzahl an RASFF-Meldungen zum Übergang von Metallionen bei Lebensmittelkontaktmaterialien aus Glas zeigt, dass ein Gesundheitsrisiko von Erzeugnissen aus (dekoriertem) Glas ausgehen kann. Zugleich weist sowohl die geringe Anzahl der meldenden Mitgliedstaaten als auch die Anwendung nationaler Beurteilungsgrundlagen auf den Bedarf einer europäischen Entscheidungsgrundlage für den Übergang von $\mathrm{Pb}$ und $\mathrm{Cd}$ bei Erzeugnissen aus Glas hin. Während die Rahmen-VO lediglich allgemeine Anforderungen an Lebensmittelkontaktmaterialien formuliert, gelten die Bestimmungen der Keramik-RL ausschließlich für Lebensmittelkontaktmaterialien aus Keramik. Weiterhin schließt der technische Leitfaden zu Metallen und Legierungen CM/Res(2013)9 des Europäischen Direktorates für die Qualität von Arzneimitteln (EDQM) Lebensmittelkontaktmaterialien aus Glas oder Keramik ausdrücklich aus dem Anwendungsbereich aus. Zugleich bemängelt auch das Bundesinstitut für Risikobewertung (BfR) die aus toxikologischer Sicht nicht mehr aktuellen Grenzwerte der bereits 1984 in Kraft getretenen und zuletzt 2005 geänderten Keramik-RL. In seiner aktualisierten Stellungnahme Nr. 023/2005 vom 26. März 2004 empfiehlt das BfR eine Verschärfung der europäischen Beurteilungsgrundlage. Aufgrund neuer toxikologischer Daten befindet sich die Stellungnahme derzeit in Überarbeitung (BfR 2017).

In der Working-Group des Standing Committee on Plants, Animals, Food and Feed (SCoPAFF) werden die in der Keramik-RL definierten Grenzwerte für den Übergang von $\mathrm{Pb}$ und $\mathrm{Cd}$ bei Lebensmittelkontaktmaterialien aus Keramik bereits seit 2011 hinterfragt. Darüber hinaus wurde die Berücksichtigung anderer Metallionen sowie eine Ausweitung des Anwendungsbereichs auf Lebensmittelkontaktmaterialien aus Glas und Emaille diskutiert. Ebenso fanden weitere Aspekte wie Grenzwerte für den Übergang von Metallionen aus dem Trinkrand Berücksichtigung. Gegenwärtig erarbeitet die Europäische Kommission einen Entwurf für eine Verordnung (European Commission 2017a).

\subsection{Migration von primären aromatischen Aminen bei Lebensmittelkontaktmaterialien}

Hinsichtlich der Meldungen zur Migration von paA bei Lebensmittelkontaktmaterialien aus China oder Hongkong ist mutmaßlich die VO (EU) Nr. 284/2011 von Relevanz, welche die EU-Kommission bereits im März 2011 aufgrund häufiger Verstöße gegen europäische Vorschriften erlassen hat. Basierend auf der Europäischen Kontroll-VO (Verordnung (EG) Nr. 882/2004) beinhaltet die Rechtsnorm 
spezielle Einfuhrbedingungen für Kunststoffküchenartikel aus Polyamid und Melamin mit Herkunft aus China oder Hongkong. Demnach gilt für diese Lebensmittelkontaktmaterialien sowohl eine Mitführpflicht von Konformitätserklärungen und Laborberichten mit Analyseergebnissen als auch eine Vorführpflicht der Erzeugnisse bei Einführung in die Union. Dabei werden Dokumentenprüfungen bei allen eingehenden Sendungen, Nämlichkeitskontrollen und Warenuntersuchungen bei $10 \%$ der eingehenden Sendungen durchgeführt. Trotz erhöhter Anforderungen lässt sich ein vergleichsweise hoher Anteil an Meldungen zur Migration bei Lebensmittelkontaktmaterialien aus Kunststoff oder Nylon mit dem Ursprung China oder Hongkong feststellen. Demnach ließ sich bislang durch die Maßnahme der EU-Kommission keine vollständige Vermeidung solcher Warnmeldungen erzielen.

In den Untersuchungen wurde eine Migration von paA bei Lebensmittelkontaktmaterialien in beträchtlichen Konzentrationen mit deutlichen Überschreitungen der definierten Nachweisgrenze festgestellt. Besonders bedenklich erscheint die Migration von paA angesichts der potenziell karzinogenen Wirkung einzelner Substanzen. Die CLP-Verordnung (Verordnung (EG) Nr. 1272/2008) über die Einstufung, Kennzeichnung und Verpackung von Stoffen und Gemischen führt 4,4'-MDA, 4,4'-Bi-o-toluidin sowie TDA als Humankanzerogene der Kategorie 1B auf und benennt diese Vertreter der paA als wahrscheinlich karzinogen beim Menschen.

Bereits in der Stellungnahme Nr. 021/2014 vom 24. Juli 2013 „Primäre aromatische Amine aus bedruckten Lebensmittelbedarfsgegenständen “ formulierte das BfR eine Handlungsempfehlung zur Verschärfung der Beurteilungsgrundlage für die Migration von paA bei Lebensmittelkontaktmaterialien. Für Substanzen, welche in der CLPVerordnung als Humankanzerogen der Kategorie 1A oder 1B eingestuft werden, schlägt das BfR die Anwendung des ALARA-Prinzips (As Low As Reasonable Achievable) und eine Herabsetzung der in der Kunststoff-VO definierten Nachweisgrenze von $0,01 \mathrm{mg} / \mathrm{kg}$ auf $0,002 \mathrm{mg} / \mathrm{kg}$ vor. Nach wiederholter Diskussion in Sitzungen der BfR-Kommission für Bedarfsgegenstände (BfR 2013, 2014, 2015a) wurde die Nachweisgrenze von $0,002 \mathrm{mg} / \mathrm{kg}$ für karzinogene paA bereits in die BfR-Empfehlung IX. (BfR 2015b), LI. (BfR 2015c) und XXXVI. (BfR 2015d) zu Materialien für den Lebensmittelkontakt übernommen.

\subsection{Migration von Ausgangsstoffen der Kunststoffherstellung bei Lebensmittelkontaktmaterialien}

Der hohe Anteil an Warnmeldungen zur Migration von Ausgangsstoffen der Kunststoffherstellung bei Schraubdeckelgläsern (13 von 37 Meldungen; 35,1\%) ist mutmaßlich auf die europäische Kampagne „Migration von Weichmachern aus Deckeldichtungen von Glasgefäßen in ölige Lebensmittel“ zurückzuführen, in deren Rahmen schwerpunktmäßig Schraubdeckelgläser auf die Migration von Ausgangsstoffen untersucht wurden (McCombie et al. 2012).

In den vorliegenden Warnmeldungen wird die Migration von Melamin anhand von 2 unterschiedlichen Grenzwerten beurteilt. Während Meldungen aus dem Jahr 2012 auf einem Grenzwert von $30 \mathrm{mg} / \mathrm{kg}$ beruhen, wurde die Migration von Melamin seit 2013 ab einem Grenzwert von $2,5 \mathrm{mg} / \mathrm{kg}$ beanstandet. Diese deutliche Herabsetzung des in der Kunststoff-VO (EU) Nr. 10/2011 definierten Grenzwerts erfolgte Ende 2011. Zuvor veröffentlichte die European Food Safety Authority (EFSA) im April 2010 ein umfassendes wissenschaftliches Gutachten zu Melamin. In der Publikation definierte die europäische Behörde einen TDI-Wert (tolerable daily intake) von $0,2 \mathrm{mg} / \mathrm{kg}$ Körpergewicht/Tag und führte Lebensmittelkontaktmaterialien auch bei Einhaltung des Grenzwertes von $30 \mathrm{mg} / \mathrm{kg}$ als potenzielles Gesundheitsrisiko für Kinder an. Ungeachtet einer Herabsetzung Ende 2011 wurde der geänderte Grenzwert von 2,5 mg/kg erst ab dem Jahr 2013 angewandt. Erwähnenswert scheinen in diesem Zusammenhang die deutlich geringeren Messwerte bei Warnmeldungen $a b$ 2013. Diese sind womöglich mit einer in Anbetracht des geänderten Grenzwerts optimierten Produktqualität zu begründen.

Aufgrund des Inkrafttretens der VO (EU) Nr. 284/2011 mit verstärkten Kontrollen ab Juli 2011 erscheint ebenso die Entwicklung der Warnmeldungen interessant. Demnach ließe sich die vergleichsweise hohe Anzahl an Meldungen im Jahr 2012 womöglich auf die intensivierten Einfuhrkontrollen zurückführen, während die ab 2013 abnehmende Anzahl an Meldungen als gesteigertes Risikobewusstsein der Hersteller und Importeure sowie daraus resultierende positive Effekte auf die Produktqualität ausgelegt werden könnte. Allerdings wurde im Jahr 2017 ein erneuter Anstieg der Warnmeldungen zur Migration von Ausgangsstoffen der Kunststoffherstellung verzeichnet. Im Jahr 2012 übermittelte die Kontaktstelle von Deutschland 4 der 7 Meldungen, darüber hinaus wurden 4 der 6 Meldungen im Jahr 2017 von Belgien veröffentlicht. Zumindest für das Jahr 2012 lässt sich die Beobachtung womöglich auf eine verstärkte Überwachung der „Freisetzung von Formaldehyd aus Bedarfsgegenständen mit Lebensmittelkontakt aus Melamin“ als Programmbestandteil des Bundesweiten Überwachungsplanes (BÜp) im Jahr 2012 zurückführen (BVL 2013). Ungeachtet der verschärften Einfuhrbedingungen befanden sich unter den Warnmeldungen zu Lebensmittelkontaktmaterialien aus Melaminharz jährlich 2 Meldungen zu Lebensmittelkontaktmaterialien mit Herkunft aus China. 
Zuletzt stieg diese Anzahl auf 4 Warnmeldungen im Jahr 2017. Um die Entwicklung der RASFF-Meldungen im Hinblick auf die VO (EU) Nr. 284/2011 beurteilen zu können, wäre eine längerfristige Untersuchung der RASFFMeldungen bereits vor dem Jahr 2012 erforderlich.

Gemäß Artikel 9 der VO (EU) Nr. 284/2011 sind Mitgliedstaaten $\mathrm{zu}$ einer quartalsweisen Berichterstattung an die EU-Kommission verpflichtet. Ein Bericht soll - neben allgemeinen Informationen zu eingegangenen Sendungen die Ergebnisse der Kontrollen sowie die Häufigkeit weiterführender Nämlichkeitskontrollen und Warenuntersuchungen beinhalten. Nach offiziellen Leitlinien zu der VO (EU) Nr. 284/2011 soll die Berichterstattung der Europäischen Kommission und den Mitgliedstaaten dazu dienen, einerseits die Konformität ebendieser Kunststoffküchenartikel $\mathrm{zu}$ überwachen und andererseits die Wirksamkeit der Maßnahmen regelmäßig zu überprüfen (European Commission 2011). Zum gegenwärtigen Zeitpunkt liegen keine offiziellen Informationen der Europäischen Kommission über die Wirksamkeit der Maßnahmen nach VO (EU) Nr. 284/2011 vor. Zuletzt stellte die Europäische Kommission eine umfassende Evaluation des geltenden europäischen Rechtsrahmens für Lebensmittelkontaktmaterialien in Aussicht. In welchem Umfang die ab dem ersten Quartal 2018 vorgesehene Evaluation Einzelmaßnahmen und Sondervorschriften wie die VO (EU) Nr. 284/2011 berücksichtigt, ist bislang nicht ersichtlich (European Commission 2017b).

\section{Schlussfolgerungen}

In den Jahren 2012 bis 2017 wurden im Europäischen Schnellwarnsystem RASFF 164 Warnmeldungen zu Lebensmittelkontaktmaterialien übermittelt. Anhand der Meldungen informierten sich die Kontaktstellen der Mitgliedstaaten über drei wesentliche Gesundheitsrisiken: Den Übergang von Metallionen (54 von 164 Meldungen; $32,9 \%)$, die Migration von primären aromatischen Aminen (50 von 164 Meldungen; 30,5\%) und die Migration von Ausgangsstoffen der Kunststoffherstellung (37 von 164 Meldungen; 22,6\%). Innerhalb dieser Kategorien lassen sich wiederum Auffälligkeiten bei Art und Material der betroffenen Lebensmittelkontaktmaterialien sowie den zugrundeliegenden Gesundheitsrisiken feststellen.

Ein Übergang von Metallionen ließ sich vornehmlich bei Utensilien zur Darreichung und Aufnahme von Lebensmitteln wie Gläsern, Bechern, Tassen und Geschirr aus (dekoriertem) Glas oder Keramik (mit Dekoration oder Glasur) nachweisen. In diesem Kontext identifizierten die zuständigen Labore häufig einen gleichzeitigen Übergang von $\mathrm{Pb}$ und $\mathrm{Cd}$ aus dem Trinkrand von Gläsern, Bechern, Tassen aus (dekoriertem) Glas. Mangels einer europäischen Beurteilungsgrundlage beanstandeten die meldenden Mitgliedstaaten diesen Sachverhalt zumeist unter Verwendung nationaler Normen.

Die Migration von primären aromatischen Aminen wurde häufig bei Utensilien zur Zubereitung wie Pfannenwendern sowie Utensilien zur Darreichung und Aufnahme von Lebensmitteln wie Servierlöffeln aus Kunststoff oder Nylon identifiziert. Im Rahmen der Migrationsuntersuchungen ließen sich humankanzerogene Substanzen wie 4,4'-Methylendianilin in beträchtlichen Konzentrationen feststellen, für welche das BfR bereits seit einigen Jahren eine Herabsetzung der Nachweisgrenze und somit des Grenzwerts fordert.

Eine Migration von Ausgangsstoffen der Kunststoffherstellung insbesondere bei Utensilien zur Darreichung und Aufnahme von Lebensmitteln wie Geschirr aus Melaminharz erwies sich ebenfalls als häufiger Gegenstand von Warnmeldungen. Diese ließen sich vor allem auf die Ausgangsstoffe Melamin und Formaldehyd zurückführen, welche mitunter in hohen Konzentrationen nachgewiesen wurden.

Mit der Verordnung (EU) Nr. 284/2011 erließ die EUKommission bereits ab Juli 2011 spezielle Einfuhrbedingungen für Kunststoffküchenartikel aus Polyamid oder Melamin aus China oder Hongkong. Dennoch stammen Lebensmittelkontaktmaterialien, bei denen eine Migration von primären aromatischen Aminen aus Kunststoff und Nylon oder eine Migration von Ausgangsstoffen der Kunststoffherstellung bei Erzeugnissen aus Melaminharz nachgewiesen wurde, häufig aus China - wobei in diesem Zusammenhang ausschließlich Warnmeldungen $\mathrm{zu}$ bereits in der Union befindlichen Artikeln berücksichtigt werden, nicht aber Grenzzurückweisungsmeldungen zu einer verweigerten Einfuhr. Demnach wäre eine längerfristige Untersuchung potenzieller Effekte unter Berücksichtigung der Warnmeldungen, Informations- und Grenzzurückweisungsmeldungen im RASFF zur Einschätzung der Wirksamkeit dieser Maßnahme von Interesse.

Die Analyse der Warnmeldungen zu Lebensmittelkontaktmaterialien lässt 3 wesentliche Gesundheitsgefahren erkennen: Den Übergang von Metallionen, die Migration von primären aromatischen Aminen und die Migration von Ausgangsstoffen der Kunststoffherstellung. Schwerpunkte bei Art und Material betroffener Produkte sowie der ursächlichen Substanz bieten Ansatzpunkte für gezielte Maßnahmen. Für einzelne Materialien und Substanzen liegen bereits Grenzwerte vor, an anderer Stelle wiederum zeigt sich anhand dieser Analyse ein Bedarf an einer adäquaten europäischen Rechtsgrundlage.

Open Access This article is distributed under the terms of the Creative Commons Attribution 4.0 International License (http://creative commons.org/licenses/by/4.0/), which permits unrestricted use, 
distribution, and reproduction in any medium, provided you give appropriate credit to the original author(s) and the source, provide a link to the Creative Commons license, and indicate if changes were made.

\section{Referenzen}

BfR (2013) 11. Sitzung der BfR-Kommission für Bedarfsgegenstände. Protokoll vom 12. November 2013. www.bfr.bund. de. Accessed 22 May 2018

BfR (2014) 13. Sitzung der BfR-Kommission für Bedarfsgegenstände. Protokoll vom 12. November 2014. www.bfr.bund. de. Accessed 22 May 2018

BfR (2015a) 14. Sitzung der BfR-Kommission für Bedarfsgegenstände. Protokoll vom 15. April 2015 www.bfr.bund.de. Accessed 22 May 2018

BfR (2015b) BfR-Empfehlung IX. Farbmittel zum Einfärben von Kunststoffen und anderen Polymeren für Bedarfsgegenstände. www.bfr.ble.de. Accessed 22 May 2018

BfR (2015c) BfR-Empfehlung LI. Temperaturbeständige Beschichtungssysteme aus Polymeren für Brat-, Koch- und Backgeräte. www.bfr.ble.de. Accessed 22 May 2018

BfR (2015d) BfR-Empfehlung XXXVI. Papiere, Kartons und Pappen für den Lebensmittelkontakt. www.bfr.ble.de. Accessed 22 May 2018

BfR (2017) Blei und Cadmium aus Keramik. Aktualisierte Stellungnahme* Nr. 023/2005 des BfR vom 26. März 2004. www.bfr. bund.de. Accessed 22 May 2018

BMEL (Ed.) (2016) Lebensmittelsicherheit verstehen. Fakten und Hintergründe. Stand Januar 2016. www.bmel.de/publikationen. Accessed 22 May 2018

BVL (Hrsg.) (2013) Berichte zur Lebensmittelsicherheit 2012. Bundesweiter Überwachungsplan 2012. Gemeinsamer Bericht des Bundes und der Länder. Springer. https://doi.org/10.1007/ 978-3-319-02810-1
European Commission (2009) The Rapid Alert System for Food and Feed (RASFF). 30 years of keeping consumers safe. Office for Official Publications of the European Communities. https://doi. org/10.2772/10448

European Commission (2011) EU guidelines on conditions and procedures for the import of polyamide and melamine kitchenware originating in or consigned from People's Republic of China and Hong Kong Special Administrative Region, China. Version 1. https://ec.europa.eu/food/sites/food/files/safety/docs/ cs_fcm_legis_china_guidelines_import-polymide-melamine.pdf. Accessed 22 May 2018

European Commission (2017a) FCM MS WG Group Ceramic. 27 November 2017. http://ec.europa.eu/food/sites/food/files/safety/ docs/cs_fcm_ceramic_presentation.pdf. Accessed 22 May 2018

European Commission (2017b) Evaluation of legislation on food contact materials-regulation (EC) No 1935/2004. Evaluation and fitness check roadmap: Ares(2017)5809429. https://ec. europa.eu/info/law/better-regulation/initiatives/ares-20175809429/feedback/add_en. Accessed 22 May 2018

European Commission (o.J.) RASFF-Food and feed safety alerts. http://ec.europa.eu/food/safety/rasff. Accessed 22 May 2018

McCombie G, Harling-Vollmer A, Morandini M, Schmäschke G, Pechstein S, Altkofer W, Biedermann M, Biedermann-Brem S, Zurfluh M, Sutter G, Landis M, Grob K (2012) Migration of plasticizers from the gaskets of lids into oily food in glass jars: a European enforcement campaign. Eur Food Res Technol 235:129-137. https://doi.org/10.1007/s00217-012-1739-2

Peltzer MA, Beldi G, Jakubowska N, Simoneau C (2015) Scoping investigations on the release of metals from the rim area of decorated articles. In: Support of the revision of the ceramics directive 84/500/EEC. JRC science and policy report. Publications Office of the European Union. https://doi.org/10.2788/ 484454 